\title{
Vascular Complications during Ilizarov Fixator Surgery to the Femur. Two Case Reports with the Introduction of a Method for Determining the Safe Vascular Corridor Around the Femur
}

Ashraf A Khanfour ${ }^{1}$, Ayman A Khanfour ${ }^{2}$

\begin{abstract}
Aim: To perform an in-depth review of the safe vascular corridor around the femur in order to decrease possible vascular injury. Background: Despite regular use of half pins in the femur, there is no region entirely safe for percutaneous pin placement. Damage to a major nerve or vessel must be avoided at all costs. Vascular complications during llizarov fixator surgery to the femur are rare but serious.

Case description: Of 306 cases of Ilizarov fixation to the femur in the period from 2002 to 2016, two cases had vascular complications. The first case developed a delayed superficial femoral artery (SFA) pseudoaneurysm and the second case sustained an early deep femoral artery (SFA) injury. Conclusion: The in-depth review of the vascular anatomy around the femur with relevance to the placement of half pins indicates that the femoral shaft segment greatest at risk to lead to a vascular injury lies between the two points: 8 and $24 \mathrm{~cm}$ proximal to the adductor tubercle. Keywords: Femur, Femoral artery, llizarov, Pseudoaneurysm, Safe corridor.

Strategies in Trauma and Limb Reconstruction (2019): 10.5005/jp-journals-10080-1434
\end{abstract}

\section{BACKGROUND}

Vascular injuries after llizarov fixator applications to the femur have been reported but are uncommon. There are six publications: four case reports, one letter to editor in English literature, ${ }^{1-3}$ and one case report in a Portuguese ${ }^{4}$ publication.

From 2002 to 2016, 306 cases were operated on using the Ilizarov method to the femur. Several pathologies were treated, e.g., for fractures, nonunion, deformity, limb length discrepancy, and techniques involving segmental bone transport, knee arthrodesis, and hip joint arthrodiatasis used. Two cases were identified to have sustained vascular injuries after llizarov fixator application. The first presented late as a pseudoaneurysm formation of the superficial femoral artery (SFA). The second presented as an early postoperative bleed from a deep femoral artery (DFA) territory 14 days after surgery.

The aim of this work is to carry out an in-depth review the safe vascular corridor around the femur to decrease the incidence of possible iatrogenic vascular injuries during half pin insertion to the femur.

\section{Case Descriptions}

The study was approved by the local ethical committee (General Organization of Teaching Hospitals and Institutes Research Ethical Committee) and was conducted in accordance with the Declaration of Helsinki and Ethical Guidelines for Epidemiological Research (2008).

\section{Case 1}

A 57-year-old male presented with a pathological fracture of the upper one-third left femur after radical saucerization as treatment of chronic osteomyelitis of the femur (Fig. 1). There was a past history of previous interventions in the form of pus drainage, sequestrectomy, guttering, and multiple debridement. Open reduction, iliac bone grafting, and llizarov external fixation were carried out. Ambulation
${ }^{1}$ Department of Orthopedic Surgery, Damanhour Medical National Institute, Damanhour, Egypt

${ }^{2}$ Department of Anatomy, Faculty of Medicine, Alexandria University, Alexandria, Egypt

Corresponding Author: Ashraf A Khanfour, Department of Orthopedic Surgery, Damanhour Medical National Institute, Damanhour, Egypt, Phone: +2/01003311476, e-mail: Dr_ashrafkhanfour@hotmail.com

How to cite this article: Khanfour AA, Khanfour AA. Vascular Complications during Ilizarov Fixator Surgery to the Femur. Two Case Reports with the Introduction of a Method for Determining the Safe Vascular Corridor Around the Femur. Strategies Trauma Limb Reconstr 2019;14(2):106-110.

Source of support: Nil

Conflict of interest: None

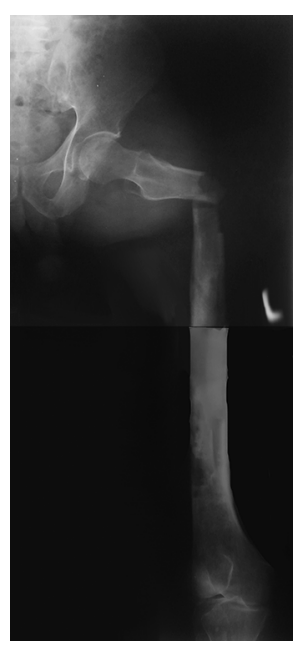

Fig. 1: X-ray of the left femur with evidence of chronic osteomyelitis and a pathological fracture 
commenced on the 7th postoperative day. In the 5 th postoperative month, the patient started to complain from a neuralgic pain along the anteromedial aspect of the leg and foot. At that time, X-rays showed ongoing union (Fig. 2). In the 8 th postoperative month, the patient noticed a nonpulsatile painful swelling increasing on the anteromedial aspect of his left thigh (Fig. 3). Computed tomography angiography revealed a sizable $(16 \times 10 \times 19 \mathrm{~cm})$ pseudoaneurysm of the SFA close to the tip of the fifth Schanz pin (distal to proximal in Fig. 4). At that time, the fractured had united. The llizarov fixator was removed under supervision of vascular surgeons in the operative theater (Fig. 5) and vascular reconstruction carried out subsequently.

\section{Case 2}

A 24-year-old male sustained a highly comminuted opened supracondylar fracture of the right femur from a motor cycle accident. Percutaneous stabilisation was done using an Ilizarov external fixator. When weight bearing was commenced 14 days postoperatively, he started complain of bleeding around the Schanz pins proximal to the fracture site. This required a readmission to the hospital and blood transfusion. Computed tomography

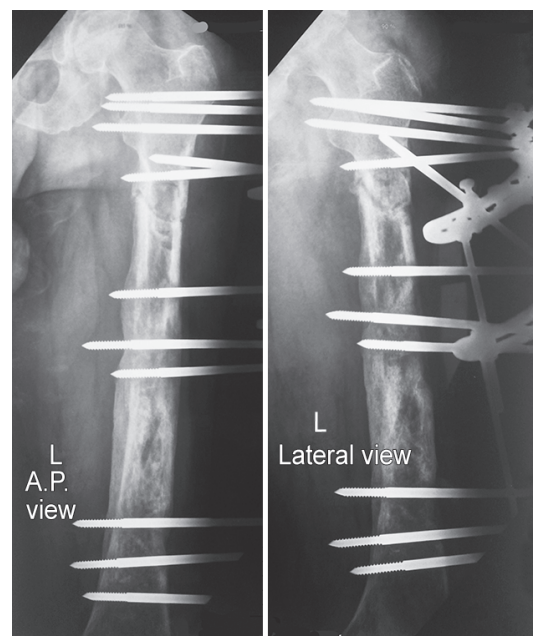

Fig. 2: X-rays of the left femur at the 5th month of follow-up showing ongoing union. There is overpenetration of the half-pins especially the fifth one from distal angiography done (Fig. 6) showed a pseudoaneurysm of one of the mid-thigh perforators of the DFA from the tip of a Schanz pin. Vascular surgeons were consulted and an exploration through a medial incision was performed with stay suturing and ligation of the damaged perforator. The fracture united by the 9 th month and the fixator was removed.

\section{Discussion}

There is no region of the femur that is entirely safe for percutaneous pin fixation and injury to a major nerve or vessel must be avoided at all costs. ${ }^{5-7}$ Injury to the femoral artery during Ilizarov fixator application is rare but serious enough to warrant an analysis in an attempt to avoid recurrence.

We were able to identify overdrilling far beyond the medial femoral cortex at the adductor canal (AC) as the cause of the SFA pseudoaneurysm in case 1 . The level at which this injury occurred (the fifth Schanz pin from distal to proximal on the AP X-ray) will be elaborated on later. Another potential cause is chronic irritation from the protruding sharp tip that is characteristic of the design of Schanz pins that may have caused delayed perforation to the SFA wall. Of note, in this area, is the close proximity of the saphenous nerve to the SFA at this site, ${ }^{8}$ which explains the pain described by the patient. The delayed diagnosis of the pseudoaneurysm is owing to a slow increase in size to the point of detection. ${ }^{9}$

Case 2 highlights the importance of accurate pin insertion away from the area of the DFA, perforator femoral arteries (PFAs), and the longitudinal arterial channel located posteromedially on the femoral shaft.

Anatomically, femoral arteries are in intimate relationship to the medial aspect of the femoral shaft; so, having a thorough knowledge of its surgical anatomy is mandatory for prevention of iatrogenic injury. ${ }^{6,7}$ Topographically, the common femoral artery (CFA) and its subsequent continuation, the SFA, are represented on the upper two-third of a drawn line running from the midinguinal point to the femoral adductor tubercle (AT) while the thigh is slightly flexed, abducted, and laterally rotated. The CFA starts subcutaneously under the mid-inguinal point anterior to the hip joint. After take-off of the DFA, approximately $4 \mathrm{~cm}$ distal to the mid-inguinal point, it continues subcutaneously as the SFA in the femoral triangle where at its apex, it enters the $A C$ in which it passes about $6 \mathrm{~cm}$ in length in a straight medial position and
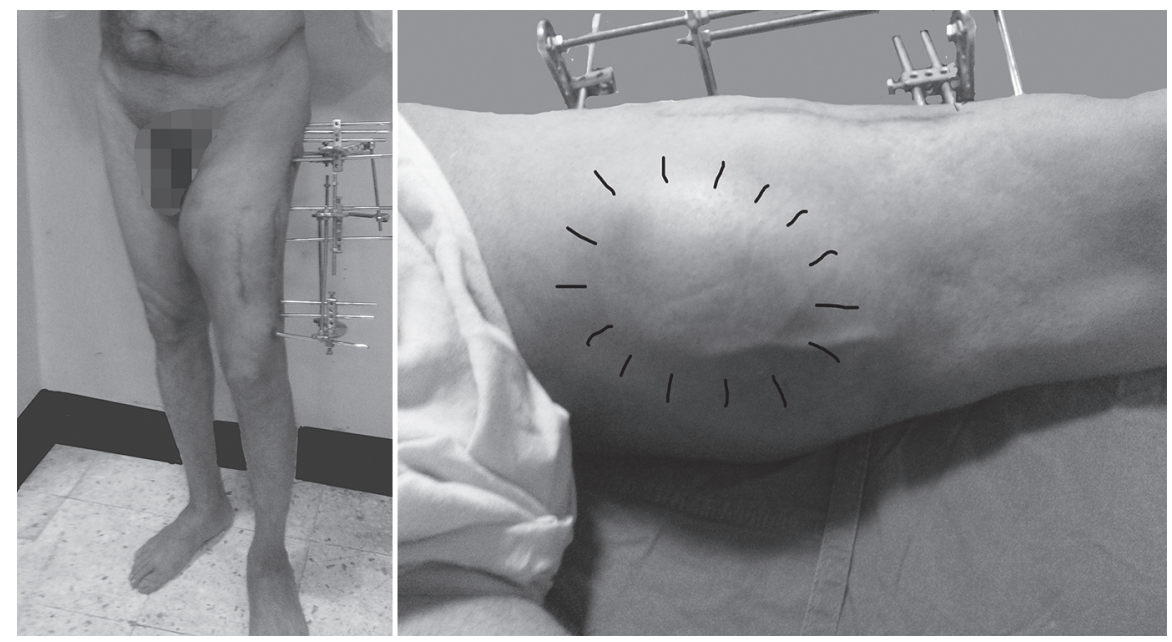

Fig. 3: A large swelling on the anteromedial aspect of the left thigh 
ends at the adductor hiatus $(\mathrm{AH})$ located $8-12 \mathrm{~cm}$ proximal to the AT where it dips posteriorly to continue as the popliteal artery. $5,10,11$ On the contrary, the DFA runs, being the main blood supply to the thigh, together with its perforators on a deep course in close relation to the medial cortex of the upper femur. It originates from the lateral aspect of the CFA about $4 \mathrm{~cm}$ distal to the mid-inguinal point, then winds inferiorly and deeply in posterolateral direction away from the SFA where it lies in the groove between psoas and pectineus muscles just medial to the subtrochanteric area of the femur. It is about $30 \mathrm{~cm}$ long. The first part, about $12 \mathrm{~cm}$, descends sandwiched between the adductor longus anteriorly and the brevis posteriorly. The second part (about $5 \mathrm{~cm}$ ) continues this descent sandwiched between vastus medialis anteriorly and adductor magnus posteriorly. Last, the third part (about $13 \mathrm{~cm}$ ) starts at the apex of the femoral triangle where it lies posterior to the SFA and then continues its descent near to the linea aspera of the femur (the "asperal part"12,13). Three perforating arteries

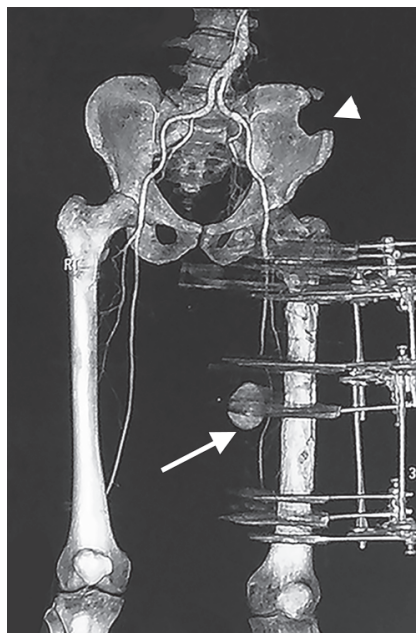

Fig. 4: CT angiography reveals a large $(16 \times 10 \times 19 \mathrm{~cm})$ pseudoaneurysm of the superficial femoral artery (SFA) close to an external fixator pin (white arrow) at the site of the AC with extensive mural thrombus. The SFA lumen is compressed but still patent at $7 \mathrm{~mm}$ and the neck of the pseudoaneurysm is $4 \mathrm{~mm}$. Left iliac bone graft donor site is seen (white arrowhead)
(PFAs) branch from the DFA while it is anterior to the adductor brevis muscle, the first originates above, the second anterior, and the third below that muscle. All the three penetrate through the adductor magnus near its attachment to the linea aspera. After its perforation to the posterior compartment of the thigh, each vessel gives ascending and descending branches, which interconnect to form a longitudinal arterial channel that also lies directly on the posteromedial aspect of the femoral shaft (Fig. 7). Then the DFA terminates as the fourth perforating artery that pierces the adductor magnus muscle variably proximal to the level of its $\mathrm{AH} .{ }^{14}$ These perforators undergo many anatomical variations that makes their topographic landmarks unreliable. ${ }^{12,13,15}$

This classic anatomic description of the vascular anatomy of the thigh does not suffice for a safe placement of percutaneous pins. ${ }^{16}$ This prompted several authors to carry out either radiological studies using three-dimensional computed angiography ${ }^{6,17}$ or anatomical studies using different metric rules on dissected cadaveric lower limb preparations $s^{11,16,18-21}$ to appraise the spatial relationship between femoral arteries and the medial aspect of the femur at indexed levels with defining a reliable safe corridor of the femur for pin insertion.

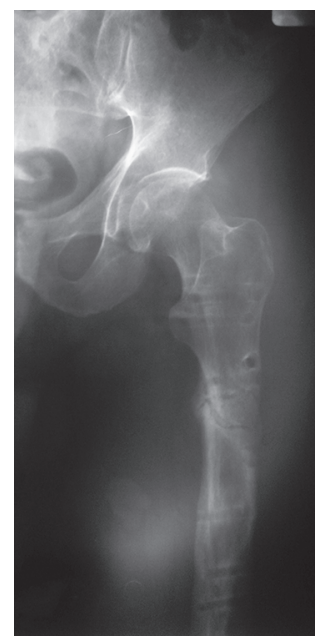

Fig. 5: X-ray of the left femur after full union of the fracture and extraction of the llizarov fixator
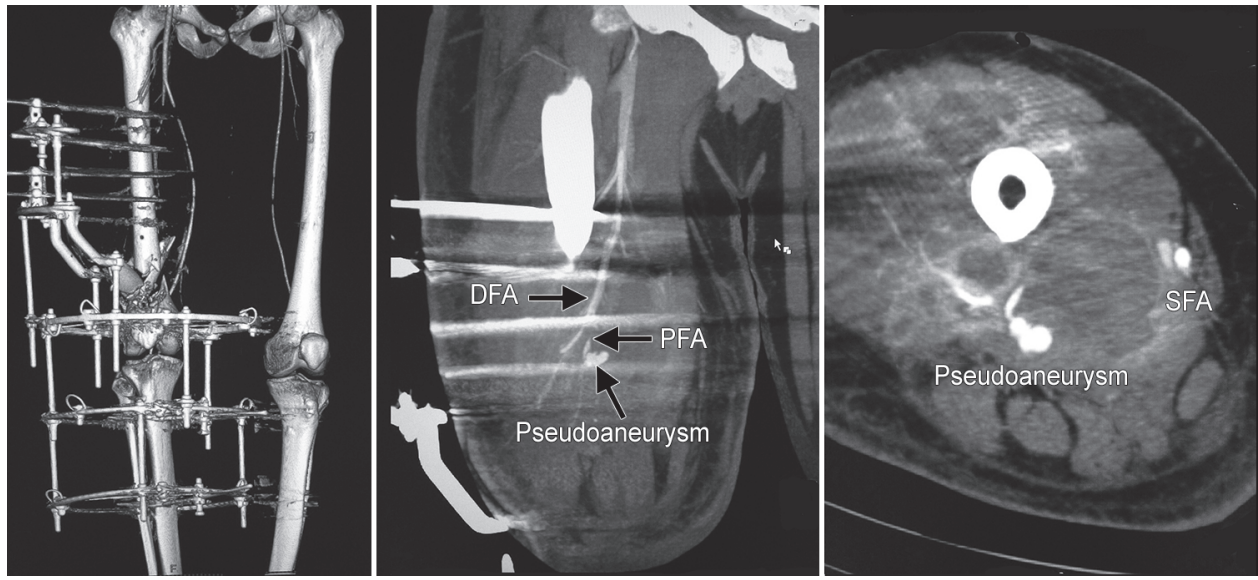

Fig. 6: A CT angiogram of the right femoral vessels showing focal injury of one of the mid-thigh perforators of the deep femoral artery with a related pseudoaneurysm averaging $3 \times 2 \mathrm{~cm}$. Leakage from this pseudoaneurysm resulted in a $18 \times 11 \times 8 \mathrm{~cm}$ hematoma with enhancing walls that splayed the superficial femoral artery 


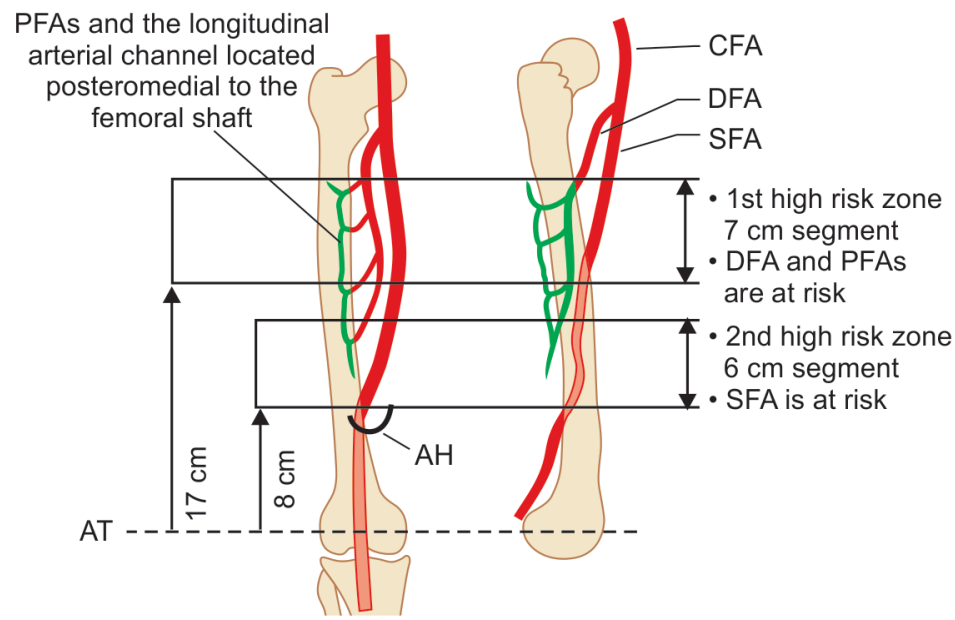

Fig. 7: A diagram demonstrating the anatomy of the common femoral artery, superficial femoral artery, deep femoral artery, perforator femoral arteries, and the longitudinal vascular channel in relation to the femur. The two high-risk zones for pin fixation to the femur are shown

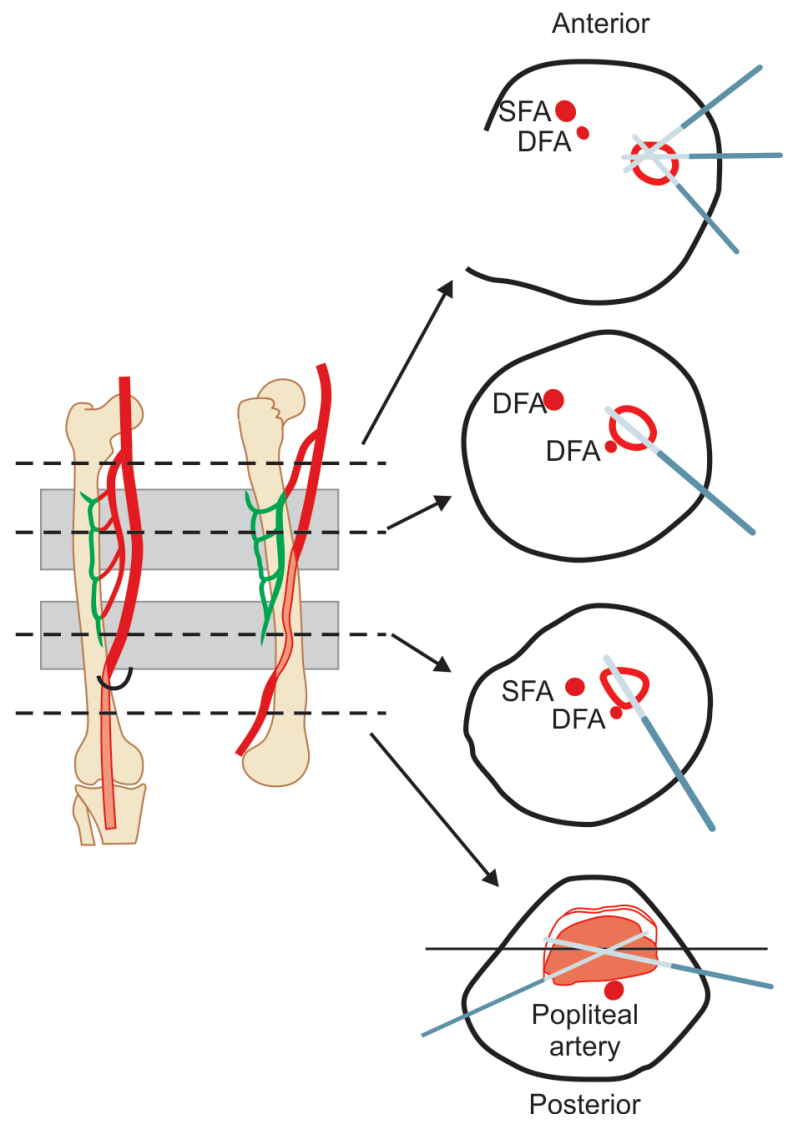

Fig. 8: A diagram demonstrating the spatial relationship of the common femoral artery, superficial femoral artery, and deep femoral artery to the defined femoral segments with recommended pin direction

Beltran et al. in 2012 concluded that the entire lateral femur could be considered safe for placing lateral or anterolateral half pins. ${ }^{18}$ This was incorrect as (1) the DFA, PFAs, and their longitudinal arterial channel lie directly on the posteromedial aspect of the femur so that pin overdrilling from the anterolateral direction would increase the risk of injury to these vessels; ${ }^{14}(2)$ the last $6 \mathrm{~cm}$ of the SFA is located inside the AC in a straight and fixed course near the medial femoral cortex making it vulnerable to be injured by overdrilled pins inserted from lateral (Fig. 7); ${ }^{19}$ and (3) the SFA runs a spatial course around the femur where it begins anterior to the femoral shaft proximally, then swipes in a posteromedial direction in the sagittal plane to become medial to the middle two-fourths of the femoral shaft (Fig. 7). 11,12,17,20

Based on a review of the anatomy and relevant literature, we determine two zones of high risk using the fixed bony landmark of the AT. This landmark is localised easily either clinically or by C-arm (Fig. 8). The first high-risk zone is a 7-cm segment located approximately $17 \mathrm{~cm}$ proximal to the AT where the DFA and PFAs lie about less than $1 \mathrm{~cm}$ of it. ${ }^{6,17,22,23} \mathrm{~A}$ second high-risk zone was defined to be a $6-\mathrm{cm}$ segment located approximately $8 \mathrm{~cm}$ proximal to the AT where the SFA lies in the subsartorial (adductor or Hunter's) canal. Consequently, at the first high-risk zone, both the DFA and PFAs are the much closer to the femur and can be easily injured while the SFA is at high risk of injury at the second high-risk zone. ${ }^{6,11,17,19-21}$

It may therefore be misleading to label what are usually termed "safe corridors" in femoral external fixation as described by Shyam et al. ${ }^{24}$ Several mitigating factors may render some corridors that are termed "safe" as unsafe, e.g., the two high-risk zones described earlier and tethering of thigh muscles leading to distorted anatomy and planes. Additionally, neurovascular risks may arise still during limb lengthening due to the changing relationship of the pin or wire as it tracks through the femur, potentially bringing it into contact with neurovascular structures, even though the initial insertion had been done correctly. ${ }^{16,24}$

In describing the two high-risk zones to lateral and anterolateral pin placement, we recognise the "safe" interval between the two aforementioned zones to be so short as to regard, from a practical perspective, the femoral shaft segment from 8 to 24 $\mathrm{cm}$ proximal to the AT as a zone at high risk to vascular injury especially if overdrilling occurred during pin insertion. Proximal to this segment fixation can be carried safely using half pins while distal to this segment, safe fixation can be safely done using both transfixing wires and half pins (Fig. 8). If possible, it is advisable to avoid placing pins in the femoral shaft within zone of high risk to vascular injury, thereby avoiding a danger of vessel penetration. If there are cases that mandate fixation in this zone, then care must 
be taken by (1) avoiding using sharp-tipped pins (conical blunttipped pins are safer if inserted manually after predrilling); (2) the pins are directed from posterolateral to anteromedial to avoid the SFA, DFA, PFAs, and the longitudinal arterial channel injury; and (3) pin insertion to be carried out under $\mathrm{C}$-arm screening to avoid overpenetration.

\section{Conclusion}

Accurate knowledge of the spatial relationships of the femoral arteries to the femur at different levels is important to avoid vascular complications during percutaneous insertion of half pins or wires. The femoral shaft segment lying between $8 \mathrm{~cm}$ and $24 \mathrm{~cm}$ proximal to the femoral AT is considered a high-risk zone for vascular injury from pins inserted from lateral or anterolateral. Early or delayed bleeding either from the wound or around the pins, local pain, swelling (with or without an associated palpable pulsation), altered distal pulses, anemia, or neuralgic pain along nerves known to be in close relation to the femoral vessels must raise attention to a possible vascular injury. Use of CT angiography is recommended to disclose these vascular complications.

\section{References}

1. Das SR, Agrawal N, Tendolkar AG, et al. Femoral artery pseudoaneurythm by external fixator [letter to the editor]. Asian cardiothoracic and thoracic annals 1998;6(2):1.

2. Khoshnevis J, Sobhiyeh MR, Fallah Zavareh M. Deep femoral artery branch pseudoaneurysm after orthopedic procedure requiring surgical treatment: a case report. Trauma Mon 2012;17(2):305-308. DOI: 10.5812/traumamon.5181.

3. Polak WG, Pawlowski S, Skora J, et al. Vascular complications after the treatment with ilizarov external fixators. Vasa 2001;30(2):138-140. DOI: 10.1024/0301-1526.30.2.138.

4. Guarniero R, Aguiar ET, Montenegro NB. Vascular complications of the Ilizarov method. Rev Hosp Clin Fac Med Sao Paulo 1993;48(1): 17-21.

5. Giachino A. Anatomic considerations in the placement of percutaneous pins. In: Uhthoff HK. Current concepts of external fixation of fractures. Berlin, Heidelberg, New York: Springer-Verlag; 1982. pp. 203-206.

6. Sun Q, Fan G, Li X, et al. Relationship between femur and femoral arteries for identifying risk factors for vascular injury. Medical science monitor: international medical journal of experimental and clinical research 2017;23:1733-1740. DOI: 10.12659/msm.900252.

7. Khanfour AA, El-Sayed MM. Efficacy of a compliant semicircular llizarov pin fixator module for treating infected nonunion of the femoral diaphysis. Strategies Trauma Limb Reconstr 2014;9(2): 101-109. DOI: 10.1007/s11751-014-0199-6.
8. Kapoor R, Adhikary SD, Siefring C, et al. The saphenous nerve and its relationship to the nerve to the vastus medialis in and around the adductor canal: an anatomical study. Acta Anaesthesiol Scand 2012;56(3):365-367. DOI: 10.1111/j.1399-6576.2011.02645.x.

9. Tiwary SK, Kumar S, Khanna R, et al. latrogenic femoral artery aneurysms in orthopaedic practice. ANZ J Surg 2007;77(10):899-901. DOI: 10.1111/j.1445-2197.2007.04269.x.

10. Shoja MM. Girdle, gluteal region and thigh. In: Standring S. Gray's Anatomy, the Anatomical Basis of Clinical Practice. 41th ed., London: Elsevier Limited; 2016. pp. 1337-1375.

11. Kale A, Gayretli O, Ozturk A, et al. Classification and localization of the adductor hiatus: a cadaver study. Balkan Med J 2012;29(4):395-400. DOI: 10.5152/balkanmedj.2012.030.

12. Lumsden AB, Colborn GL, Skandalakis LJ, et al. Surgical anatomy of the deep femoral. Artery., In: Dongen V, Dusmet M. Surgery of the Deep Femoral Artery. Berlin, Heidelberg: Springer Berlin Heidelberg; 1994. pp. 1-21.

13. Siddharth $\mathrm{P}$, Smith $\mathrm{NL}$, Mason RA, et al. Variational anatomy of the deep femoral artery. Anat Rec 1985;212(2):206-209. DOI: 10.1002/ ar.1092120216.

14. Drake RL, Vogl AW, Mitchell AWM. Gray's anatomy for students. 2nd ed., Philadelphia: Churchill livingstone Elservier; 2010. pp. 528-646.

15. Farouk O, Krettek C, Miclau T, et al. The topography of the perforating vessels of the deep femoral artery. Clin Orthop Relat Res 1999(368):255-259. DOI: 10.1097/00003086-199911000-00031.

16. Louis R, Jouve JL, Borrione F. Anatomic factors in the femoral implantation of the llizarov external fixator. Surg Radiol Anat 1987;9(1):5-11. DOI: 10.1007/bf02116848.

17. Narulla RS, Kanawati AJ. Safe zone for the superficial femoral artery demonstrated on computed tomography angiography. Injury 2016;47(3):748-751. DOI: 10.1016/j.injury.2015.12.012.

18. Beltran MJ, Collinge CA, Patzkowski JC, et al. The safe zone for external fixator pins in the femur. J Orthop Trauma 2012;26(11):643-647. DOI: 10.1097/BOT.0b013e31824aed95.

19. Olson SA, Holt BT. Anatomy of the medial distal femur: a study of the adductor hiatus. J Orthop Trauma 1995;9(1):63-65. DOI: 10.1097/00005131-199502000-00010.

20. Checroun AJ, Mekhail AO, Ebraheim NA, et al. Extensile medial approach to the femur. J Orthop Trauma 1996;10(7):481-486. DOI: 10.1097/00005131-199610000-00006.

21. Kwon JY, Johnson CE, Appleton $P$, et al. Lateral femoral traction pin entry: risk to the femoral artery and other medial neurovascular structures. J Orthop Surg Res 2010;5(1):4. DOI: 10.1186/1749-799X-5-4.

22. Catagni MA, Maiocchi AB. Atlas for the insertion of transosseous wires and half-pins: ilizarov method. 2nd revised edition ed., Milan-Italy: Medi Surgical Video; 2003.

23. Sabharwal S, Kishan S, Behrens F. Principles of external fixation of the femur. Am J Orthop (Belle Mead NJ) 2005;34(5):218-223.

24. Shyam K, Sanjeev S, Fred B, et al. External fixation of the femur: Basic Concepts. Techniques in Orthopaedics 2002;17(2):239-244. DOI: 10.1097/00013611-200206000-00013. 